\title{
Applying Particle Swarm Optimization and Differential Evolution Combined with Finite Element Method to Optimize Cable
}

\author{
Zhipeng Jiang, Xishan Wen, Xiaoqing Yuan \\ School of Electrical Engineering \\ Wuhan University \\ Wuhan, China \\ hudieyutanglang@163.com
}

\begin{abstract}
Particle swarm optimization (PSO) and differential evolution (DE) algorithm are biologically inspired computing, which simulate the behavior of survival of the fittest from the systems of nature biology, ecology and so on. As heuristic random algorithms, they have characteristics of self-adaptation, self-organization, selflearning, etc. PSO and DE can be used to solve various complex problems that are difficult by using traditional calculation methods. Based on the platform of ANSYS finite element soft, parametric programming of a single-phase cable and a three-phase cable is accomplished in the paper by using ANSYS parameter design language (APDL). Optimization calculation of the maximum electric field strength of cable insulation layer is realized by PSO and DE combined with finite element method (FEM). That biologically inspired computing combined with FEM provides reference and enlightenment for optimizing highvoltage equipment in aspects of electromagnetic field.
\end{abstract}

Keywords-particle swarm optimization; differential evoluti on algorithm; finite element method; cable; optimization

\section{INTRODUCTION}

In recent decades, bionic intelligence algorithms for solving complex optimization problems by simulating natural ecosystems and organisms mechanism have been put forward and studied, such as genetic algorithm, ant colony algorithm, particle swarm optimization (PSO), artificial bee colony algorithm, differential evolution (DE) algorithm, artificial immune algorithm, DNA algorithm, etc. These bionic intelligence algorithms greatly enrich modern optimization technique, show powerful vitality and development potential in solving complex optimization problems and engineering application [1-6]. Last few years, bionic intelligence algorithms have an increasingly wide range of application research in power system that involves power network planning, reactive power optimization, economic load dispatch, optimal power flow calculation, device structure optimization and other aspects. At present, since finite element method (FEM) has become as one of the main methods on electromagnetic field numerical calculation in power system, the size of calculation model parameters can be changed by parametric finite element program based on the optimal object, moreover, the versatility and the flexibility of parametric finite element program is particularly suited for high-voltage device optimization. Bionic intelligence algorithms combined with FEM is gradually showing wide application prospect in electrical equipment structure optimization, such as the authors in paper [7] put forward genetic algorithm combined with FEM to optimize multiple structural parameters of grading rings for composite insulator that succeeded in gaining satisfied effect.

In the paper, based on previous studies, the structure optimization of a single-phase cable and a three-phase cable on electrical field numerical calculation is implemented by PSO and DE combined with FEM by using ANSYS parameter design language (APDL). The maximum electric field strength inside the single-phase cable and the three-phase cable is as optimal objective function during the optimal process, finite element parametric programming for the single-phase cable and the three-phase cable is accomplished, which is combined with two bionic intelligence algorithms by using APDL. Furthermore, the analysis on the results of cable optimization with PSO and DE combined with FEM is completed in this paper. The paper provides reference and inspiration for structure optimization of high-voltage device in application of bionic intelligence algorithms combined with FEM.

\section{CABLE DESIGN PROBLEM}

\section{A. CABLE DESIGN}

The determination of cable thickness is one of the keys of power cable design. That the factors should be considered in the design of cable thickness include: (a) electric field distribution inside cable insulation layer; (b) withstand voltage during runtime of cable; (c) breakdown statistical law of cable insulation material; (d) mechanical strength and technological property of cable insulation material. When the working voltage of cable is more than $10 \mathrm{kV}$, the breakdown strength of cable insulation gradually becomes the main factor of cable thickness [8].

\section{B. CABLE MODEL}

In the paper, simplified models of the single-phase cable and the three-phase cable [9] are taken as research objects. There is an assumption for the coaxial cable that the coaxial cable is composed of conductor and surrounding cable insulation layer without considering 
conductor shield, insulation shield, air gap, corrugated aluminum sleeve and so on. The structure diagram of the single-phase cable is as shown in Figure 1(a), $R_{E}$ represents the diameter of cable insulation layer, $\mathrm{R}_{\mathrm{C}}$ represents the diameter of conductor. The maximum field strength $E_{\max }$ inside the single-phase cable insulation layer is present on the surface of the inner conductor. There is an analytical expression for $E_{\max }$ showing in equation (1). Figure 1(b) illustrates the geometry of the three-phase cable. The minimum value of the maximum field strength $\mathrm{E}_{\max }$ inside the three-phase cable insulation layer is an optimization problem with bivariate parameters that include the conductor diameter $\mathrm{R}_{\mathrm{C}}$ and another parameter radius $\mathrm{R}_{\mathrm{I}}$. In the electric field optimization problem of the cables, the maximum field strength $E_{\max }$ inside cable insulation layer can be used as individual fitness value function to evaluate perfection degree of individuals during the optimization process.

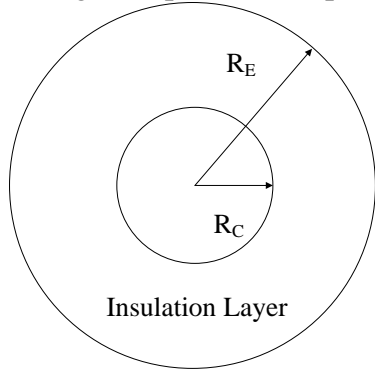

(a) Single-phase

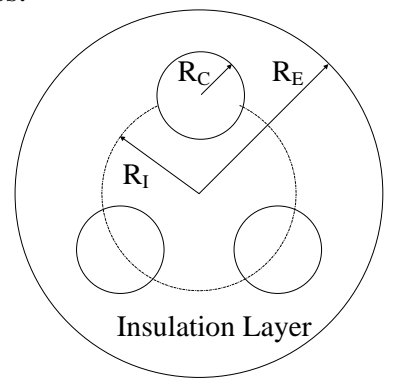

(b) Three-phase
Figure 1. The structure diagram of cable

The value of $E_{\max }$ in the single-phase cable depends on the ratio between $R_{E}$ and $R_{C}$, and it is as follows:

$$
E_{\text {max }}=U /\left(R_{C} \cdot \ln \left(R_{E} / R_{C}\right)\right)
$$

In equation [1], $U$ is loading voltage of the conductor in the single-phase cable.

Since the length dimension of cable model is much larger than the radial dimension of it, the electric field component along the length direction of cable can be considered as zero with ignoring the end effect, and then the electric field numerical calculation of cable can adopt two-dimensional plane model of FEM. Normalization processing is made for the structure parameters of the single-phase cable and the three-phase cable. Set $\mathrm{R}_{\mathrm{E}}=1$ p.u., $\mathrm{U}=1$ p.u., $\mathrm{R}_{\mathrm{C}}$ is as optimization objective, when $\mathrm{R}_{\mathrm{C}}=1 / \mathrm{e}=0.3679$ p.u., the minimum value of $\mathrm{E}_{\max }$ is equal to 2.7183 p.u.. The loading voltages of the three conductors in the three-phase cable are sequentially: 1 p.u., -0.5 p.u., 0.5 p.u.

\section{OPTIMIZATION ALGORITHMS}

\section{A. PSO and DE}

PSO and DE both are heuristic and randomly bionic intelligence algorithms. Their similarities are that the population is initialized by random function and individual selection is implemented by greedy mode. While their differences is their evolutionary modes that population regeneration of PSO and DE are achieved by speed and displacement matrix equation, mutation and crossover operation respectively.

\section{B. Algorithm Flow}

In this paper, the general steps of optimization process for cable based on PSO and DE combined with FEM are as follows:

Step1: Initializing the population by random function with APDL at first.

Step2: Evaluating the initial population by FEM in ANSYS software.

Step3: Regenerating population of PSO by speed and displacement matrix equation, while regenerating population of DE by mutation and crossover operation.

Step4: Handling renewal population based on constraints.

Step5: Selecting individual by greedy mode.

Step6: If the convergence criterion is satisfied exit, the process will be terminated, else go to step 3 .

The optimization parameters of PSO that are individual learning coefficient $\mathrm{C}_{1}$ and group learning coefficient $\mathrm{C}_{2}$ that are both 2.1, the parameters of DE that are scale factor $\mathrm{F}$ and crossing constant CR are equal to 0.6 and 0.9 respectively in the paper. In the optimization process of the single-phase cable, the search range of optimal parameter $\mathrm{R}_{\mathrm{C}}$ is from 0.1 p.u. to 0.9 p.u.. In the optimization process of the three-phase cable, the search range of optimal parameter $\mathrm{R}_{\mathrm{I}}$ is from 0.42 p.u. to 0.68 p.u., while that of optimal parameter $\mathrm{R}_{\mathrm{c}}$ is from 0.05 p.u. to 0.35 p.u.

\section{OPTIMIZATION RESULTS}

\section{A. Results of the Single-Phase Cable}

The optimization parameters of the single-phase cable that are population iteration number and population size are 25 and 10 respectively. Evolution curves of $E_{\max }$ by PSO and DE combined with FEM are as shown in Figure 2 , evolution curves of $R_{C}$ can be seen from Figure 3 . And optimum parameters are shown in Table I.

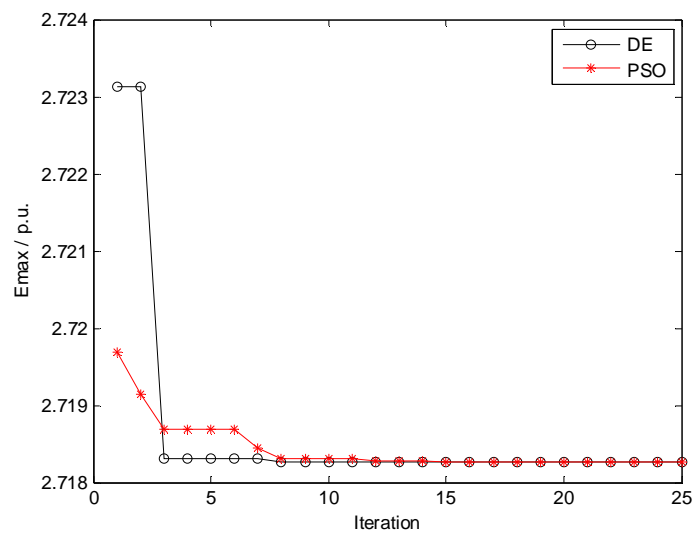

Figure 2. Evolution curves of $\mathrm{E}_{\max }$ 


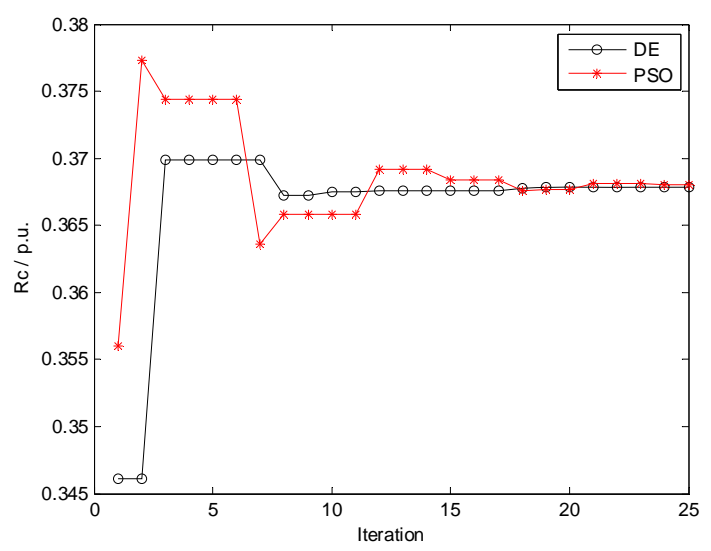

Figure 3. Evolution curves of $\mathrm{R}_{\mathrm{C}}$

TABLE I. Optimization Results of The Single-Phase CABle

\begin{tabular}{|c|c|c|c|}
\hline Optimum Parameters & Analytical Value & DE & PSO \\
\hline $\mathrm{R}_{\mathrm{C}} /$ p.u. & 0.3679 & 0.3679 & 0.3680 \\
\hline $\mathrm{E}_{\max } /$ p.u. & 2.7183 & 2.7183 & 2.7183 \\
\hline
\end{tabular}

Optimum parameter of $\mathrm{R}_{\mathrm{C}}$ by $\mathrm{DE}$ is equal to 0.3679 p.u.. Figure 4 and figure 5 preset potential nephogram and electric field nephogram of the single-phase cable by DE in optimum parameter respectively.

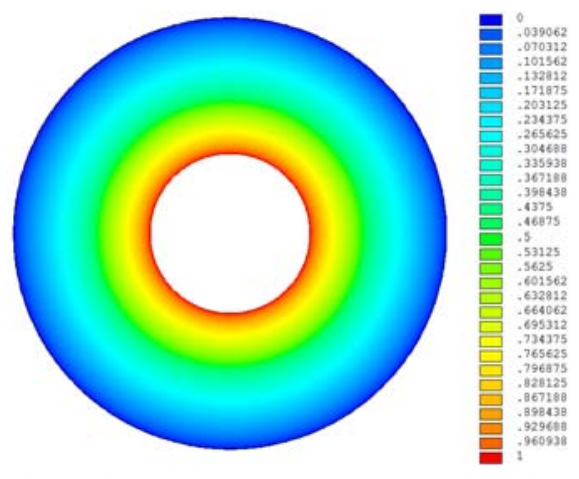

Figure 4. Potential nephogram of the single-phase cable

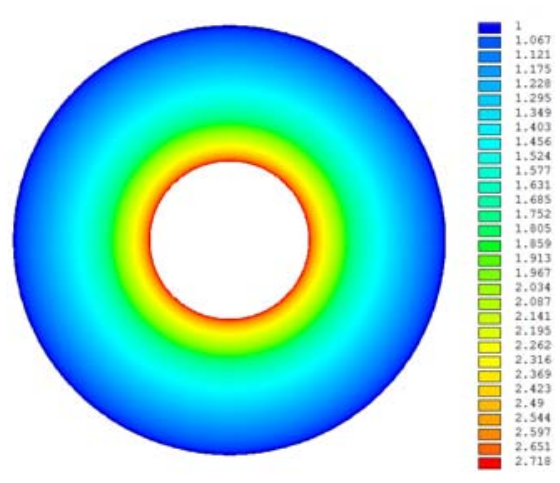

Figure 5. Electric field nephogram of the single-phase cable
From the above results, it can be seen early convergence rate of PSO is better than that of DE in the optimization process of the single-phase cable, optimum parameters of $\mathrm{E}_{\max }$ are both converged to the analytical value, optimum parameter of $\mathrm{R}_{\mathrm{C}}$ by $\mathrm{DE}$ is better than that by PSO.

\section{B. Results of the Three-Phase Cable}

Population iteration number and population size in the optimization process of the three-phase cable are equal to 50 and 10 respectively. Evolution curves of $E_{\max }$ are as shown in Figure 6, evolution curves of $\mathrm{R}_{\mathrm{I}}$ can be seen from Figure 7. Optimum parameters are shown in Table 2. Figure 8 shows evolution curves of $\mathrm{R}_{\mathrm{C}}$. Reference values of $\mathrm{R}_{\mathrm{C}}$ and $\mathrm{R}_{\mathrm{I}}$ in paper [9] are 0.178 p.u. and 0.510 p.u. respectively.

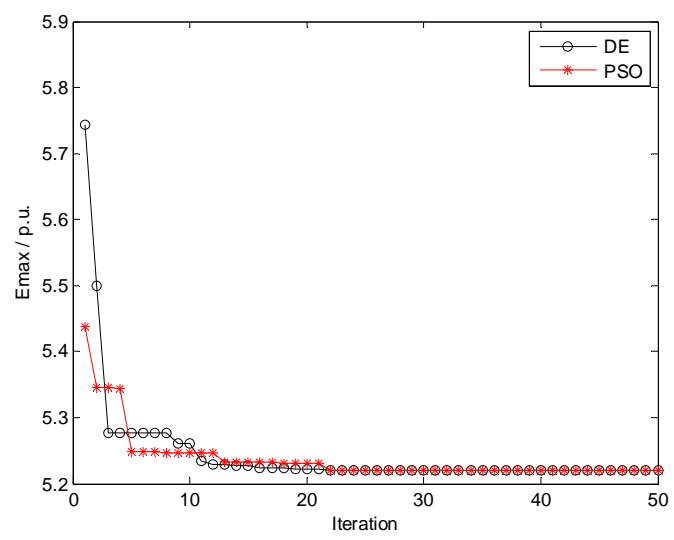

Figure 6. Evolution curves of $\mathrm{E}_{\max }$

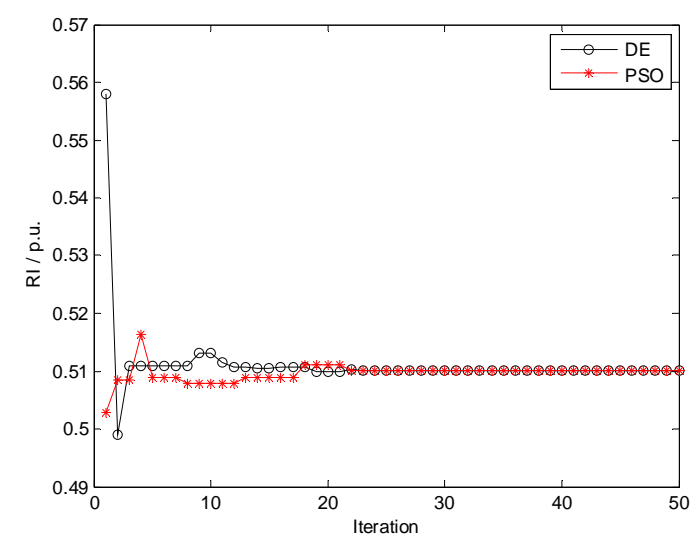

Figure 7. Evolution curves of $\mathrm{R}_{\mathrm{I}}$

TABLE II. OPtimizATION Results OF THE THREE-PHASE CABLE

\begin{tabular}{|c|c|c|c|}
\hline Optimum Parameter & Paper[9] & DE & PSO \\
\hline $\mathrm{R}_{\mathrm{C}} /$ p.u. & 0.178 & 0.1761 & 0.1762 \\
\hline $\mathrm{R}_{\mathrm{I}} /$ p.u. & 0.510 & 0.5101 & 0.5102 \\
\hline $\mathrm{E}_{\max } /$ p.u. & $/$ & 5.2195 & 5.2196 \\
\hline
\end{tabular}




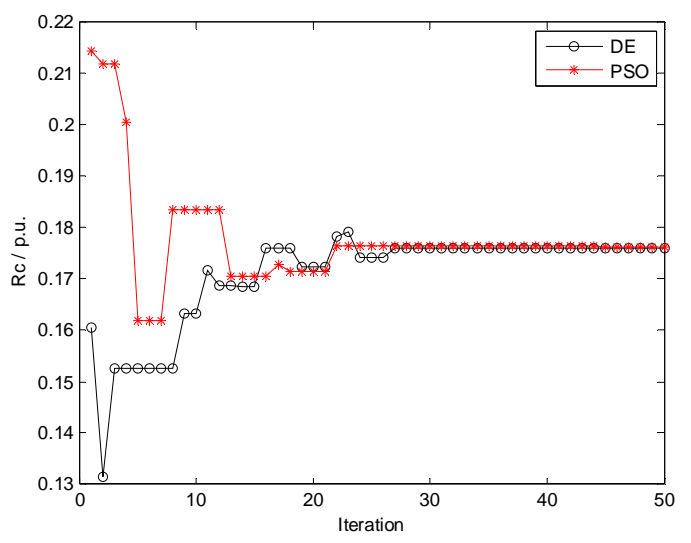

Figure 8. Evolution curves of $\mathrm{R}_{\mathrm{C}}$

Optimum parameters of $\mathrm{R}_{\mathrm{C}}$ and $\mathrm{R}_{\mathrm{I}}$ by PSO are equal to 0.1762 p.u., 0.5102 p.u.. Figure 9 shows potential nephogram of the three-phase cable by PSO in optimum parameters, and figure 10 represents electric field nephogram of it by PSO in optimum parameters.

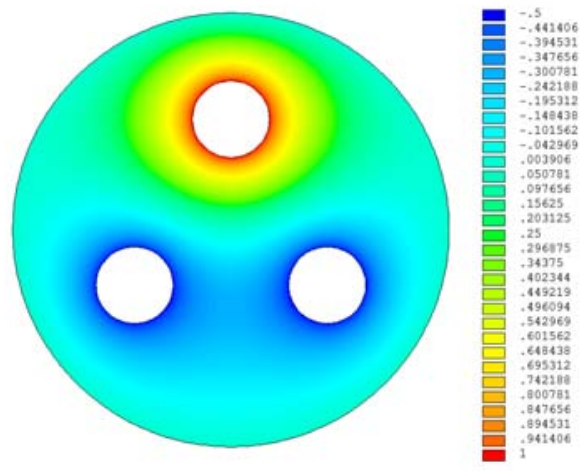

Figure 9. Potential nephogram of the three-phase cable

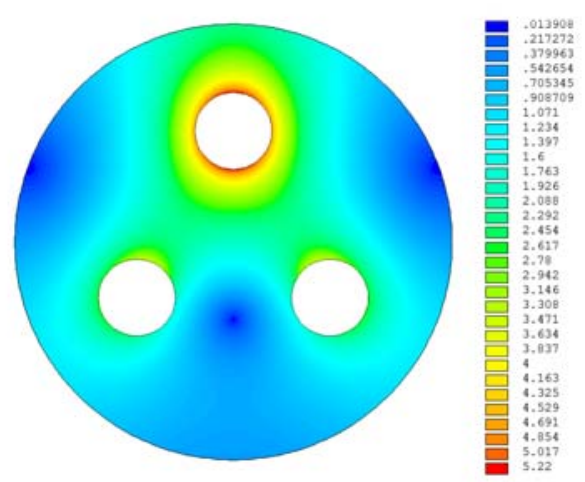

Figure 10. Electric field nephogram of the three-phase cable

From Figure 6 to figure 10 and table 2, it can be seen that optimum parameters of $\mathrm{R}_{\mathrm{I}}$ based on PSO and DE are very close to reference value, and relative errors that $R_{C}$ between reference value and optimum value by PSO and
$\mathrm{R}_{\mathrm{C}}$ between reference value and optimum value by ED are $1.01 \%$ and $1.07 \%$ respectively. And optimum result of $\mathrm{E}_{\max }$ by DE with FEM is better than that by PSO with FEM.

\section{CONCLUTION}

In this study, comparing PSO with DE in optimization of the single-phase and the three-phase cable by using APDL on the platform of ANSYS finite element software, the performance of PSO is better than that of DE at previous search process, while optimum result of DE is better than that of PSO. PSO and DE combined with FEM are general and can be used to optimize not only on cable, but also on any other power system apparatus. Whereas integrating and absorbing the respective advantages of PSO and DE in order to make them learn from each other is very significant for engineering optimization calculation based on FEM, and it is also the focus of the follow-up study of the authors.

\section{REFERENCES}

[1] H. B. Duan, X. Y. Zhang, C. F. Xu. Bio-inspired computing. Beijing: Science Press, 2005.

[2] J. Kennedy, R. Eberhart. Particle swarm optimization. Proceedings of IEEE Internation Conference on Neural Networks, 1995: 19421948.

[3] R. Eberhart, J. Kennedy. A new optimizer using particle swarm theory. Proceedings of the 6th International Symposium on MicroMachine and Human Science, 1995: 39-43.

[4] M. Clerc, J. Kenndey. The particel swarm: Explosion, stability, and convergence in a multidimensional complex space[J]. IEEE Transactions on Evolutionary Compution, 2002, 6(1):58-73.

[5] R. Storn, K. Price. Differential evolution—a simple and efficient adaptive scheme for global optimization over continuous spaces. Berkley: International Computer Science Institute, 1995.

[6] K. Price. Differential evolution a fast and simple numerical optimizer. Proceedings of Biennial Conference of the North American Fuzzy Information Processing Society, 1996: 524-527.

[7] L. Huang, X. S. Wen, L Lan, et al. Optimization of grading rings for UHV composite insulator by the improved GA[J]. High Voltage Engineering, 2009, 35(2): 218-224.

[8] J. Y. ZHOU. Principle of power cable design. Beijing: China Machine Press, 1999.

[9] W. Sima, F. P. Espino-Cortes, E. A. Cherney, etc. Optimization of corona ring design for long-rod insulators using FEM based computational analysis[C]. in: Electrical Insulation, 2004. Conference Record of the 2004 IEEE International Symposium on: 2004. 480-483.

[10] http://en.wikipedia.prg/wiki/Bio-inspired-computing.

[11] J. Holland. Adaptation in natural and artificial systems. Cambridge: The MIT Press,1975. 